Editorial

\title{
Acknowledgement to Reviewers of Journal of Composites Science in 2018
}

\author{
Journal of Composites Science Editorial Office
}

MDPI, St. Alban-Anlage 66, 4052 Basel, Switzerland

Published: 9 January 2019

Rigorous peer-review is the corner-stone of high-quality academic publishing. The editorial team greatly appreciates the reviewers who contributed their knowledge and expertise to the journal's editorial process over the past 12 months. In 2018, a total of 71 papers were published in the journal, with a median time to first decision of 14 days and a median time to publication of 40 days. The editors would like to express their sincere gratitude to the following reviewers for their cooperation and dedication in 2018:

\author{
Abbasi, Hooman \\ Abdelkarim, Omar \\ Abisset-Chavanne, Emmanuelle \\ Ahn, Byungmin \\ Aitomäki, Yvonne \\ Alderliesten, René \\ Altan, M. Cengiz \\ Amaechi, Chiemela Victor \\ Ansari, Farhan \\ Arbelaiz, Aitor \\ Artero Guerrero, Jose Alfonso \\ Asadi, Amir \\ Assih, Jules Tchao \\ Auclair, Nicolas \\ Bacigalupo, Andrea \\ Balandin, Alexander \\ Balle, Frank \\ Baranowska-Bosiacka, Irena \\ Barretta, Raffaele \\ Barszczewska-Rybarek, Izabela \\ Bayraktar, Emin \\ Benmokrane, Brahim \\ Bergmann, Jean Pierre \\ Bica, Ioan \\ Binetruy, Christophe \\ Birely, Anna C \\ Blanco, Ignazio \\ Branda, Francesco \\ Brnić, Josip \\ Brunner, Andreas \\ Buckner, Steven W. \\ Bui, Tinh Quoc
}

\author{
Burkel, Eberhard \\ Cabeza Simo, Marta Maria \\ Caminero, M.A. \\ Cao, Yuhe \\ Caporali, Stefano \\ Caputo, Francesco \\ Castro, Jose \\ Cavallaro, Giuseppe \\ Cesano, Federico \\ Chandrashekhara, K. \\ Chen, Shuo \\ Chen, Chunhui \\ Chiachio, Manuel \\ Choi, Heung Soap \\ Cicala, Gianluca \\ Compton, Brett G. \\ Cooney, Ralph \\ Czaderski, Christoph \\ Davoudinejad, Ali \\ De Moura, Marcelo Francisco De Sousa Ferreira \\ Di Girolamo, Rocco \\ Di Sarno, Luigi \\ DiLandro, Luca \\ Dimitri, Rossana \\ Dong, Chensong \\ Dumont, Pierre J.J. \\ Faghih Shojaei, Mostafa \\ Fatyeyeva, Kateryna \\ Feng, Chuang \\ Fernandes, Emanuel M. \\ Fernandes, Fábio \\ Finkenstadt, Victoria
}


Forster, Aaron M.

Fukuda, Takeshi

Gafurov, Marat

Gereke, Thomas

Ghaffar, Seyed

Giorcelli, Mauro

Goh, Kheng-Lim

González, Israel

Gotsis, Alexandros D.

Groh, Rainer

Guessasma, Sofiane

Gutierrez, Junkal

Ha, Youn Doh

Hagenbeek, Michiel

Hanaor, Dorian

Harničárová, Marta

Heitzmann, Michael

Hong, Suck Won

Hooper, Paul A

Hossain, Mokarram

Huang, Chih-Feng

Huang, Ying

Imperatore, Stefania

Incarnato, Loredana

Iwamura, Munetaka

Jacob, Aboudi

Jajam, Kailash

Jajam, Kailash C.

Jezierski, Jan

Jiang, Saihua

Jiang, Ruisong

Johnston, Joel

Jopek, Hubert

Jozwik, Pawel

Kamdem, Donatien Pascal

Kang, Hyo

Kannan, Manigandan

Kärger, Luise

Khan, Arafat

Kihara, Shin-ichi

kim, Seong Su

Kim, Eunho

Kitane, Yasuo

Kotsuchibashi, Yohei

Koumoulos, Elias P.

Kvashnin, Dmitry G.

Lamuta, Caterina

Lanc, Domagoj

Lazzara, Giuseppe

Lebon, Frédéric

Lee, Jin-Kyun

Lee, Jaehong
Lekakou, Constantina

Li, Xianglin

Li, Yang

Li, Jinghao

Lima, Carmine

Linß, Sebastian

Lionetto, Francesca

Liu, Yu-Jing

Liu, Pengfei

Liu, Mingxian

Loreto, Giovanni

Luciano, Raimondo

Luong, Dzung Dinh

Luzi, Francesca

Malik, Muhammad Imran

Martins Amaro, Ana

Meenashisundaram, Ganesh Kumar

Meier, Urs

Meng, Maozhou

Merodio, Jose

Micelli, Francesco

Miklaszewski, Andrzej

Moghaddam, Hesam S.

Mohagheghian, Iman

Morselli, Davide

Muc, Aleksander

Naser, M. Z.

Naumenko, Konstantin

Odent, Jérémy

Ojovan, Michael

Orbulov, Imre

Osswald, Tim

Palumbo, Davide

Pamies, Ramon

Park, Soo-Jin

Park, Chung Hae

Pegoretti, Alessandro

Peng, Qing

Pinto, Deesy Gomes

Puglia, Debora

Rao, Agnè

Ren, Zhen

Ribeiro, Maria C.S.

Rizal, Conrad

Rodríguez Millán, Marcos

Romanowicz, Paweł

Rousakis, Theodoros

Russo, Salvatore

Ryu, Jehwang

Ryu, Sung-Hun

Saeedifar, Milad

Saimoto, Akihide 
Sain, Sunanda

Salviato, Marco

Sanz Garcia, Andres

Sazou, Dimitra

Schabowicz, Krzysztof

Scribante, Andrea

Segal, Rebecca

Shalaby, Mohamed

Shestakov, Mikhail

Shimomura, Osamu

Shirvanimoghaddam, Kamyar

Shunmugasamy, Vasanth C.

Sidorkin, Alexander

Smarzewski, Piotr

Song, Kenan

Speranzini, Emanuela

Spina, Roberto

Srinivasan, Gowrishankar

Stan, Miruna Silvia

Steup, Martin

Strankowski, Michał

Strozzi, Matteo

Summerscales, John

Szala, Mirosław

Thakur, Vijay Kumar

Todoroki, Akira

Tomota, Yo

Triantafillou, Thanasis
Trochu, François

Troiani, Enrico

Ueno, Shintaro

Umer, Rehan

Van Hemelrijck, Danny

Vincent, Thomas

Wang, Yeqing

Wang, Yongxiang

Wang, Xiao-Ming

$\mathrm{Wu}$, Xijia

$\mathrm{Xu}$, Hongyi

Yandouzi, Mohammed

Yang, Yang

Yang, Teng-Chun

Yang, An-Chih

Yeon, Jung Heum

Ying, Hanze

$\mathrm{Yu}$, Jiayi

Yu, Rena C.

Zabihi, Omid

Zeltmann, Steven Eric

Zhang, Yijie

Zhang, Hai

Zhang, Meng

Zhang, Laichang

Zhao, Wei

Zhou, Jin

(C) 2019 by the author. Licensee MDPI, Basel, Switzerland. This article is an open access article distributed under the terms and conditions of the Creative Commons Attribution (CC BY) license (http://creativecommons.org/licenses/by/4.0/). 